\title{
Unravelling complex associations between testosterone and parasite infection in the wild
}

\author{
Vanessa O. Ezenwa ${ }^{*, 1}$, L. Stefan Ekernas ${ }^{2}$ and Scott Creel $^{3}$ \\ ${ }^{1}$ Odum School of Ecology and Department of Infectious Diseases, College of Veterinary Medicine, University of \\ Georgia, Athens, Georgia 30602, USA; ${ }^{2}$ Division of Biological Sciences, University of Montana, Missoula, Montana \\ 59812, USA; and ${ }^{3}$ Department of Ecology, Montana State University, Bozeman, Montana 59717, USA
}

\section{Summary}

1. Testosterone has emerged as an important mechanism linking variation in male reproductive behaviour to parasite infection in vertebrates, and there are numerous pathways by which testosterone can influence infection risk, particularly in free-living animals.

2. The immunocompetence handicap hypothesis posits that the positive effects of testosterone on sexual signalling and behaviour are traded off with negative effects on immune function. While this obligate trade-off may sometimes explain the association between testosterone and parasite infection, testosterone-parasite relationships may also be mediated by correlated changes in stress, condition and exposure to parasites.

3. In this study, we explored associations between testosterone and reproductive behaviour, immune function and parasitism in free-living Grant's gazelle (Nanger granti). In particular, we examined how stress (glucocorticoids), behaviour and condition might mediate associations between testosterone, immunity and parasitism.

4. We found that variation in endogenous testosterone in male gazelle was correlated with mating behaviour in terms of sexual signal intensity (horn size) and resource-holding potential (territoriality). We also showed that these same levels of testosterone were associated with immunity and parasite infection, but associations between testosterone, immunity and parasitism were complex. Testosterone was negatively associated with adaptive immunity, but positively associated with innate immunity. Relationships between testosterone and parasitism varied for different parasites.

5. Our results suggest that the effects of testosterone on male immunity are not universally suppressive and that immunoenhancement may also occur. In addition, testosterone-parasite relationships vary depending on the parasite, most likely due to the opposing effects of testosterone on different aspects of immunity and indirect effects on parasite exposure mediated by changes in behaviour.

Key-words: behaviour, glucocorticoids, ICHH, immunity, Nanger granti, parasitism, sexual selection, territoriality

\section{Introduction}

Parasitism is considered to be a key factor that can influence and be influenced by host behaviour (Hamilton \& Zuk 1982; Moller, Dufva \& Allander 1993; Altizer et al. 2003). Nevertheless, significant gaps remain in our understanding of the mechanisms that link host behaviour to variation in parasite infection. In male vertebrates, androgens, particularly testosterone, play a central role in shaping behavioural, morphological and physiological

*Correspondence author. E-mail: vezenwa@uga.edu variation associated with alternative mating tactics, social status, dominance and ultimately reproductive success (Miles et al. 2007). Testosterone is also widely considered to come with fitness costs in terms of increased male mortality, due in part to a combination of direct and indirect effects of testosterone on immune function and parasite exposure (Muehlenbein \& Bribiescas 2005). As such, testosterone provides a functional link between male mating behaviour and parasite infection and represents an important starting point for unravelling the pathways by which reproductive behaviour may influence infectious disease risk. 
A number of pathways could theoretically link testosterone to behaviour-associated variation in parasite infection. One of the most widely considered paths is summarized by the immunocompetence handicap hypothesis $(\mathrm{ICHH})$, which posits that testosterone, by positively affecting secondary sexual characters on the one hand, and simultaneously depressing immunity on the other, creates a physiological trade-off between the intensity of sexual signals and risk of parasite infection (Folstad \& Karter 1992). By extension, if signal intensity influences male behaviour and intrasexual selection, then reproductive behaviour and social status should be closely linked with parasitism. While a large number of studies have tested predictions of the ICHH, a meta-analysis exploring the weight of evidence in favour of its two main assumptions, that testosterone: (i) reduces immunocompetence and (ii) increases parasitism, found limited support for both (Roberts, Buchanan \& Evans 2004). Furthermore, while the predictions of the ICHH may be relatively straightforward for experimental studies, the expectations for non-experimental studies are less clear (Peters 2000). Importantly, a suite of recent empirical studies highlight situations in which the central assumptions of the ICHH are violated. First, in some cases, the immunosuppressive effects attributed to testosterone may be a consequence of correlated changes in glucocorticoid levels (GCs) and therefore may instead reflect stress-induced immunosuppression (Evans, Goldsmith \& Norris 2000; Owen-Ashley, Hasselquist \& Wingfield 2004). Second, testosterone may not have universally suppressive effects on all aspects of immune function (Hasselquist et al. 1999; Buchanan, Evans \& Goldsmith 2003), and immunoenhancement may even occur under certain conditions (Bilbo \& Nelson 2001; Ruiz et al. 2010), particularly if testosterone levels covary with resource availability or body condition (Duckworth, Mendonca \& Hill 2001; Blas et al. 2006; Roberts \& Peters 2009). Third, testosterone may act on some parasites by altering host behaviour in ways that enhance or even reduce exposure to parasites (e.g. by altering spatial activity, home range sizes and/or contact rates: Chandler et al. 1994; Denardo \& Sinervo 1994; Grear, Perkins \& Hudson 2009), with little effect on immune-mediated susceptibility to infection. Indeed, testosterone has been linked to different effects on different parasites within the same system (Fuxjager et al. 2011), and any of the factors outlined earlier could help obscure expected relationships between testosterone and parasite infection.

From the wealth of studies on this topic, it is likely that testosterone operates on parasite risk both directly and indirectly via a complex suite of interconnected pathways (Braude, Tang-Martinez \& Taylor 1999; Casto, Nolan \& Ketterson 2001; Alonso-Alvarez, Bertrand \& Sorci 2007; Alonso-Alvarez et al. 2007; Mougeot et al. 2007; Bortolotti et al. 2009). However, it is still difficult to draw conclusions about the role testosterone plays in parasite-mediated selection in the wild. This is partly because few field studies have considered natural variation in testosterone with respect to multiple behavioural, endocrine and immunological variables that potentially link male reproductive behaviour and

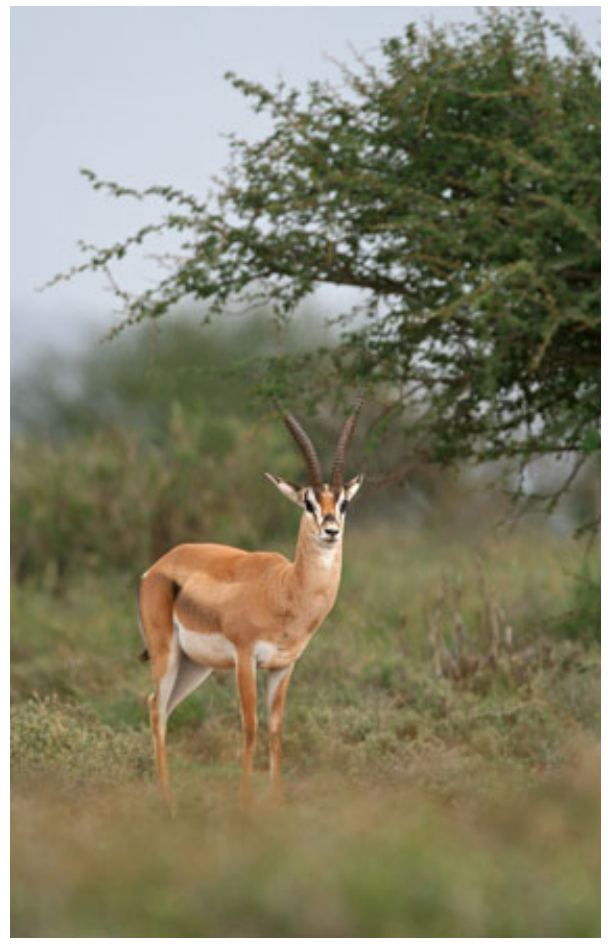

Fig. 1. Male Grant's gazelle.

parasite infection. Yet, this holistic approach applied to endogenous levels of testosterone in free-living animals is crucial for linking the results of laboratory and field-based testosterone manipulation experiments to ecologically relevant parasite-mediated fitness costs of testosterone.

In this study, we used free-living Grant's gazelle (Nanger granti; Fig. 1) as a mammalian model for evaluating potential direct and indirect links between testosterone and parasite infection risk. In Grant's gazelle, resource defence (territoriality) determines male access to females and reproductive success (Walther 1972; Walther, Mungall \& Grau 1983), and males who defend territories have been shown to have higher gastrointestinal nematode burdens than those who do not (Ezenwa 2004). To understand whether testosterone might account for such an association between male mating behaviour and parasitism, we tested for direct effects of endogenous testosterone on: (i) male mating behaviour; (ii) immune function; and (iii) parasite infection while simultaneously considering potential indirect effects mediated by GCs, condition and behaviour. Our overarching goals were to explore how testosterone-immunity/testosterone-parasite relationships might vary for different components of the immune system and different parasites and to understand the extent to which these relationships are shaped by direct vs. indirect effects.

\section{Materials and methods}

\section{ANIMALS AND SAMPLING}

We captured 35 male Grant's gazelle (N. granti) at the Mpala Research Center, Kenya $\left(0^{\circ} 17^{\prime} \mathrm{N}, 37^{\circ} 52^{\prime} \mathrm{E}\right)$, over a 6 -day period in 
August 2009. Animals were located by helicopter and captured using a hand-held net gun fired from the aircraft. Once captured, individuals were fitted with unique colour ear tags. Blood and faecal samples were collected from all animals for hormone, parasite and immunological analyses. In addition, a single observer collected information on variation in a sexually dimorphic trait (horn size), by measuring horn length (distance between the base and tip of horn on both the right and left sides), horn width (distance between horn tips) and base thickness (width of the horn at the base on both the left and right sides) for all individuals. Horn length and width were measured with a flexible plastic tape measure, and base thickness was measured using digital callipers. All measurements were made in centimetres. For horn length and base thickness, values from the right and left sides were averaged to generate a single measure of mean horn length and thickness. After sample collection and morphological measurements, animals were anesthetized to facilitate age estimation. Age was determined using tooth wear criteria for Grant's gazelle described in Stelfox, Hudson \& Groer (1985). We evaluated tooth wear in live gazelle by making an impression of the upper molar using dental putty (Provil Novo, Heraus Kulzer) and discerning wear patterns from the tooth moulds. We biased captures toward sub-adult and adult males so all males in our sample were $\geq 2.5$ years old.

Male Grant's gazelle gain access to females by defending territories on which females congregate; and territory defence in this species involves ritualized displays, aggression and sometimes direct combat (Walther, Mungall \& Grau 1983). Males that successfully acquire territories spend the majority of their time on territory, actively defend against potential competitors and herd females to keep them within the bounds of the territory (Walther, Mungall \& Grau 1983). The duration of territory ownership can vary widely across males, and males without territories join roving bachelor herds (Walther, Mungall \& Grau 1983; Ezenwa, unpublished data). At our field site, territoriality and breeding occur year round, and males can gain or lose territorial status at any time of year (Ezenwa, unpublished data). To estimate the ability of our captured males to acquire and maintain a territory, i.e. their resource-holding potential (RHP), we assessed the social status of males for 12 consecutive months following capture. We surveyed males on an approximately weekly basis between 24 August 2009 and 30 September 2010 and upon each re-sighting recorded current social status. Status was coded as territorial (T), bachelor (B) or unknown (U); territoriality was assigned based on an assessment of male behaviour, spatial location and group composition (see Fig. S1, Supporting Information). In cases where these factors could not be reliably evaluated, male status was classified as unknown. We calculated the frequency with which each male was classified as territorial over the 12-month observation period and used this as an index of resource-holding potential. To be included in the analysis, a male had to be re-sighted and classified as either T or B at least four times over the course of the observation period. We also excluded data from one 2.5-year-old male for whom a significant number of sightings were as part of a female group within another male's territory. Grant's gazelle sub-adult males usually get excluded gradually from female groups by age $\geq 2$ (Walther, Mungall \& Grau 1983), and observations of this individual suggest that it was in this transition period. Using these criteria, we calculated RHP for 20 males and the average number of re-sightings per male with definitive status classifications was 61 (range $=4-208)$.

\section{HORMONE ANALYSES}

To measure individual testosterone and glucocorticoid concentrations we collected faecal samples directly from the rectum of all ani- mals during capture. Samples were placed on ice in the field and transported to the laboratory within $10 \mathrm{~h}$ of collection where faeces was homogenized and stored in $15-\mathrm{mL}$ screwcap tubes at $-20{ }^{\circ} \mathrm{C}$ until further processing. To extract hormones from faeces, we followed the protocol of Creel, Winnie \& Christianson (2009) with slight modification. We extracted steroids from $\sim 0 \cdot 4 \mathrm{~g}$ of wet faeces by boiling in $95 \%$ ethanol for $30 \mathrm{~min}$. As we used wet samples, we corrected for variation in sample water content by calculating the per cent water in each sample. To do this, we weighed a second subsample of each faecal homogenate before (wet) and after drying (dry). Samples were dried over 3 days in a drying oven, and across all samples, average water content was $49 \cdot 1 \%($ range $=39 \cdot 8-62 \cdot 4 \%)$. We used the resulting per cent water values to convert wet sample weights to dry weights. After the boiling step, hormone extracts were dried under forced air after which tubes were sealed and transported cold to the USA, where the samples were reconstituted in $2 \mathrm{~mL}$ of $95 \%$ ethanol and stored at $-20{ }^{\circ} \mathrm{C}$ (total time before reconstitution $=5$ days). The extraction process was then completed within 30 days by evaporating off the $2 \mathrm{~mL}$ of ethanol under forced air and reconstituting samples in $1 \mathrm{~mL}$ of $100 \%$ methanol. Samples were stored at $-80{ }^{\circ} \mathrm{C}$ until hormone assays were run.

We assayed testosterone with a monoclonal antibody ELISA Assay Designs, Farmingdale, NY, USA, \#901-065), which has 14.6\% cross-reactivity to 19 -hydroxytestosterone, $7 \cdot 2 \%$ to androstendione and $<1 \%$ to other steroids (including estrogens, progestins and corticoids). A serial dilution of a pooled sample of extracts and testosterone standards showed parallelism from 29-2000 $\mathrm{pg} \mathrm{mL}^{-1}$. Sample dilutions ranged from 85 to $501: 1$ dilution, based on the testosterone concentration in the sample.

Cortisol extracts were assayed with a monoclonal antibody ELISA (Assay Designs, \#901-071) with 122\% cross-reactivity to prednisolone, $27 \cdot 7 \%$ to corticosterone and $<1 \%$ cross-reactivity to testosterone and estradiol. Serial dilution of gazelle extracts and cortisol standards were highly parallel through dilutions up to 512-fold, with an optimal dilution of $1: 30$. At a 30 -fold dilution, recovery of cortisol added to extracts was $84 \%$, and the sensitivity of the assay was an order of magnitude below the mean concentration of the diluted extracts. For biological validation of the cortisol assay, we performed an adrenocorticotropic hormone (ACTH) challenge with three captive male Grant's gazelle at the San Diego Wild Animal Park, San Diego, CA. Two males received an intramuscular injection of a $4 \mathrm{ug} \mathrm{mL} \mathrm{m}^{-1}$ ACTH gel (Optimal Compounding, Bakersfield, CA, USA), and a third male acted as a control. The challenge produced a two to fourfold increase in faecal cortisol after a 10-16 h lag.

For both testosterone and cortisol, all samples were run in duplicate, and the average of the two samples was used in analyses. If the coefficient of variation between the duplicate samples exceeded $15 \%$, samples were re-analysed. The intraplate coefficient of variation was $8.0 \%$ for testosterone and $7 \cdot 9 \%$ for the cortisol. The interplate coefficient of variation was $13 \cdot 1 \%$ for testosterone. All cortisol samples were run on a single plate. Hormone concentrations were read as pg $\mathrm{mL}^{-1}$ from the assay and expressed as $\mathrm{ng} \mathrm{g}^{-1}$ dry facces of immunoreactive faecal testosterone (fT) or faecal glucocorticoid metabolites (fGCs).

\section{IMMUNOLOGY, CONDITION AND PARASITE LOAD}

We measured two components of individual immunity - innate and adaptive immune function. Blood samples were collected from the jugular vein of individual gazelle into $10 \mathrm{~mL}$ EDTA and heparin tubes and kept on ice until transported to the laboratory. To quantify the non-cellular component of the innate immune response, we 
measured the bacteria-killing ability of plasma (Matson, Tieleman \& Klasing 2006). Plasma was harvested from heparin tubes following centrifugation and stored at $-30{ }^{\circ} \mathrm{C}$ for $7-12$ days until processing. To assay bactericidal activity, $10 \mu \mathrm{L}$ of plasma was diluted with $190 \mu \mathrm{L} \mathrm{CO}$-independent media enriched with $4 \mathrm{~mm}$ L-glutamine, and to each dilution we added a $20 \mu \mathrm{L}$ suspension of $\sim 800$ colonyforming units (CFUs) of Escherichia coli. Lyophilized E. coli pellets (EPower Microorganisms, Microbiologics, St. Cloud, MN, USA, Ref \#0483E7, ATCC \#8739) were reconstituted in phosphate-buffered saline (PBS) following the manufacturer's instructions to create a stock solution, and the stock solution was diluted daily to obtain a $\sim 800$ CFU working solution for use in assays. Stock solutions were prepared on the first and fourth day of assay runs. Once bacteria were added to plasma, samples were vortexed and incubated at $37^{\circ} \mathrm{C}$ for $30 \mathrm{~min}$. After incubation, $50 \mu \mathrm{L}$ of the plasma-bacteria mixture was plated in duplicate onto tryptic soy agar plates. Assays were performed over four consecutive days, and on each day, three plates inoculated with $50 \mu \mathrm{L}$ diluted bacteria served as positive controls, and three plates inoculated with $50 \mu \mathrm{L}$ PBS served as negative controls. All plates were incubated at $37{ }^{\circ} \mathrm{C}$, and the number of CFUs per plate was quantified after $\sim 24 \mathrm{~h}$. Because assays were performed in a field laboratory, sample preparation was carried out inside a sterile area set-up using a 25-gallon plastic storage container inverted on an aluminium foil-covered tabletop. The insides of the container and table top were swabbed daily with $95 \%$ ethanol before and after each set of assays. Bacteria-killing ability per sample was calculated as $1-\left[\left(\right.\right.$ mean $\left.\mathrm{CFU}_{\text {sample }}\right) /\left(\right.$ mean $\left.\left.\mathrm{CFU} \mathrm{U}_{\text {controls }}\right)\right]$. Bactericidal activity ranged from 10 to $73 \%(n=32$, mean $=38 \%)$, and variation in sample storage time prior to processing had no noticeable effect on killing ability (ANOva: $F_{5,26}=0 \cdot 311, P=0.902$ ).

We quantified the cellular component of innate immunity as the proportion of circulating neutrophils in peripheral blood. To do this, we made blood smears using EDTA-preserved blood within $10 \mathrm{~h}$ of blood collection, and a single observer conducted differential white blood cell counts on all smears. Counts were performed under 1000x magnification with a binocular compound microscope. As an index of standing adaptive immunity we used the proportion of circulating levels of lymphocytes from the same blood cell counts.

As an index of condition, we measured haematocrit (HCT) levels. HCT is a measure of red blood cell volume and can reflect changes in an animal's oxygen-carrying capacity and nutritional status (Delgiudice et al. 1992; Trillmich et al. 2008). To measure HCT, capillary tubes were filled with blood from heparin tubes and spun in a microhaematocrit centrifuge for $5 \mathrm{~min}$. HCT values were read off of the processed capillary tubes using a microhaematocrit reader card.

Parasite infections were assessed from faecal samples collected during capture. First, gastrointestinal nematode egg output in host faeces was quantified using a modification of the McMaster faecal egg counting technique (Ezenwa 2003a). Faecal egg counts reflect a combination of the number, size and fecundity of the worm population within a host (Gasbarre, Leighton \& Sonstegard 2001). All counts were performed on the day of sample collection. Second, we used a beaker-modified Baermann technique to quantify first-stage lungworm larvae output in faeces (Forrester \& Lankester 1997). Larvae were isolated from $10 \mathrm{~g}$ of faeces, distinguished morphologically based on body size, form and tail shape (Bowman 1999; Foreyt 2001) and enumerated with a compound microscope under 100-400× magnification. All samples were processed within 1-7 days of collection. Overall, immature stages of five distinct parasite types (Nematoda: Strongylida) were distinguished, including strongyle nematode eggs, Trichuris spp. eggs, and first-stage larvae of three distinct morphotypes, designated here as Morphs A-C. As the time of day of faecal sampling can affect parasite output (Ezenwa 2003b; Villanua et al. 2006), we evaluated whether there was an effect sampling time on parasite counts. Of the 35 animals captured, $86 \%(30 / 35)$ were caught between 07:00 and 12:00 h, while 14\% (5/35) were caught between 16:00 and 18:00 h. When we examined the effect of sampling hour on parasite counts, we found no significant effect of time on counts for any parasite $(P>0 \cdot 347)$, so we did not include sampling time as a covariate in further analyses of parasite data.

\section{STATISTICAL ANALYSES}

To investigate whether testosterone affected male horn size and resource-holding potential, and to evaluate whether there was significant covariation between testosterone, glucocorticoid and HCT levels, we tested for associations between fT, horn size, fGCs and HCT and examined whether $\mathrm{fT}$ at capture was a significant predictor of the frequency of male territoriality over a 12-month period post-capture. For each of these analyses we used a least squares regression and controlled for the effect of age. Because our three measures of horn size (width, mean length and mean thickness) were highly correlated ( $n=35$, Pearson's $r=0.68-0.89, P<0.0001$ ), we used a principle components analysis to compress these traits into a single variable representative of overall horn size. The first principle component (PC1) explained $87 \%$ of the variance in horn traits, and each individual trait was significantly correlated with PC1 (Pearson's $r \geq 0 \cdot 90$ ), so we used this as a composite measure of horn size. As horn size increased with age in a nonlinear fashion, we used a second-order polynomial regression to model the relationship between age and composite horn size and used the residuals of this model as an age-corrected measure of horn size. The residuals removed any relationship between horn size and age $(n=32$, polynomial regression, composite horn size vs. age, $r^{2}=0.53, P<0.0001$; least squares regression, residual composite horn size vs. age: $r^{2}=-0 \cdot 03$, $P=1 \cdot 0)$.

We examined the direct and indirect effects of testosterone on immune function and parasite infection by running two sets of parallel models for each immune and parasite variable. For immune function variables, we ran a first set of models testing the effect of fT alone (with age as a covariate) on dependent variables. Next, we ran full models including fGCs to account for any indirect effects of testosterone on immune function that may be mediated by glucocorticoids. For the non-cellular immunity measure (plasma bacteria killing), we included HCT in the full model to account for possible effects of condition. For the cellular immunity variables (neutrophils and lymphocytes), we included the opposing cell type in the full models, because the proportion of these two cells were negatively correlated (Spearman's rank correlation, $\left.r_{\mathrm{s}}=-0.962, P<0.0001\right)$. To evaluate the relative likelihood of our direct vs. indirect models, we used Akaike's Information Criteria (AIC). For each set of models, we calculated the AIC corrected for small sample size (AICc), the difference in AICc between each candidate model and the best fitting model ( $\triangle \mathrm{AICc}$ ), and Akaike weights. We then assessed the relative evidence in support of each model by comparing Akaike weights (Burnham \& Anderson 2002).

Because acute stress caused by animal capture and handling can influence immune function (Matson, Tieleman \& Klasing 2006), we tested whether capture and handling time was correlated with immune measures. We recorded handling time for 30 animals, and excluding a single outlier for which handling time was $66 \mathrm{~min}$, the average animal handling time was $28.5 \mathrm{~min}$ (range: 16-44). There was no significant correlation between handling time and either neutrophil or lymphocyte counts (Spearman's rank correlation, neutrophils: 
$n=29, \quad r_{\mathrm{s}}=-0.0614, \quad P=0.7516 ; \quad$ lymphocytes: $n=29$, $\left.r_{\mathrm{s}}=0.0626, P=0 \cdot 7472\right)$. However, there was trend toward a negative association between handling time and plasma bacteria killing ( $n=29$, Pearson's $r=-0 \cdot 32, P=0 \cdot 092)$. Given this, we included handling time as a covariate in all models for plasma bacteria killing. We also excluded all samples $(n=5)$ run on the third assay day from the bacteria-killing analyses because mean killing ability was significantly higher on this day compared to all other days (ANOVA, $n=32$, $\left.F_{3,28}=3 \cdot 12, P=0 \cdot 0419\right)$. The day three assay corresponded to the third day of using a single batch of bacteria stock solution, and the exclusion of samples run on this day removed any association between assay day and mean bacteria-killing activity $(n=27$, $\left.F_{2,24}=0 \cdot 117, P=0 \cdot 8898\right)$. Results of models excluding day three samples were qualitatively similar to the results of models where we included all samples and added assay 'day' as a covariate, suggesting that assay day variation did not alter our findings.

For parasite variables, we ran a first set of models testing the effect of fT alone (with age as a covariate) on dependent variables and then ran full models with fGCs to account for stress-mediated immune effects and horn size included as a proxy for possible behaviour-mediated effects. As with the immunity models, we compared the direct and indirect parasite models using Akaike weights. For all of our models, we evaluated the degree of multicollinearity among predictor variables by checking variance inflation factors (VIF). As $\mathrm{VIF}_{\text {max }}=1 \cdot 7$, we included multiple variables simultaneously in appropriate models because multicollinearity was unlikely to strongly influence model results (O'Brien 2007). We checked all variables for normality and used transformations where appropriate to normalize data distributions. fT and fGCs values were log-transformed; neutrophil and lymphocyte proportions were arcsin square root-transformed; and we $\log (x+1)$-transformed data for three parasites (strongyles, Morph B and Morph C). For analysis of transformed variables, we used least squares regressions and then tested model residuals for normality using Shapiro Wilk's tests (in all cases, $W>0.94$ and $P>0.05$ ). For analysis of a fourth parasite (Morph A), we used a negative binomial generalized linear model (GLM) as the data set best fit a negative binomial distribution. For a fifth parasite (Trichuris) that occurred at low prevalence, we used a logistic regression model (likelihood ratio test) to test the effects of fT, fGCs and horn size on infection probability. All analyses were implemented in R 2.11 ( $\mathrm{R}$ Core Development Team) or JMP 8.0.2 (SAS Institute, Inc., Cary, NC, USA). Because sample sizes vary among models because of incomplete data for some variables, we included information on sample size for each analysis reported.

\section{Results}

\section{TESTOSTERONE, HORN SIZE AND MALE RESOURCE-HOLDING POTENTIAL}

Testosterone is known to have effects on male secondary sexual traits. In male Grant's gazelle, faecal testosterone (fT) levels ranged from 90 to $453 \mathrm{ng} \mathrm{g}^{-1}$ and were positively associated with male horn size (least squares regression, $n=31, \log \mathrm{fT}$, coefficient $=2 \cdot 85, P=0.0069$; age, coefficient $=-0.05, P=0.5786$; Fig. 2a). In addition, fT was a significant and positive predictor of the frequency of territoriality (least squares regression, $n=19, \log \mathrm{fT}$, coefficient $=1.02, \quad P=0.0038 ; \quad$ age,$\quad$ coefficient $=0.006$, $P=0.8208$; Fig. 2b). Although there was a marginal and
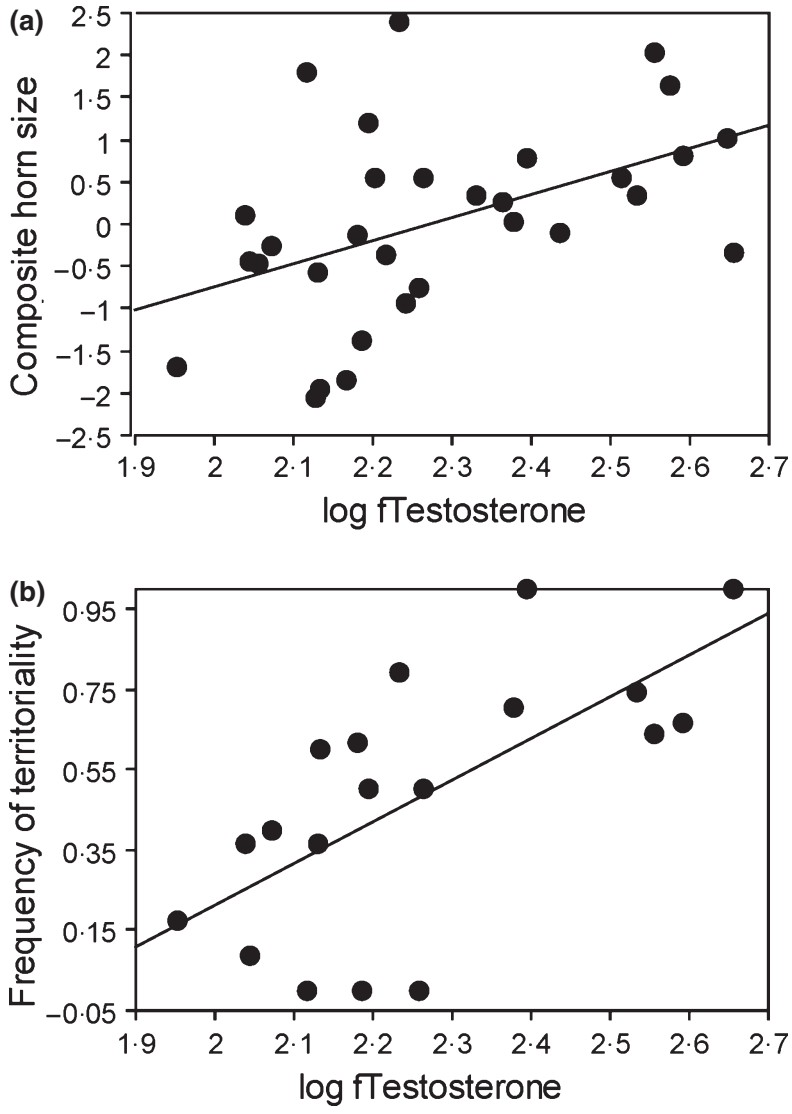

Fig. 2. Associations between faecal testosterone at the time of capture and (a) horn size, (b) frequency of territoriality over a 12-month period post-capture.

positive association between horn size and frequency of territoriality (least squares regression, $n=20$, horn size, coefficient $=0 \cdot 112, \quad P=0 \cdot 074 ;$ age, coefficient $=0 \cdot 045$, $P=0 \cdot 194)$, once $\mathrm{fT}$ was accounted for this association disappeared, and $\mathrm{fT}$ was the only significant predictor of territoriality (least squares regression, $n=19, \log \mathrm{fT}$, coefficient $=$ $0.94, P=0.0147$; horn size, coefficient $=0.03, P=0.5914$; age, coefficient $=0 \cdot 01, P=0 \cdot 7218)$.

\section{TESTOSTERONE, GLUCOCORTICOIDS AND CONDITION}

Correlated changes in glucocorticoids commonly accompany changes in male testosterone levels. Faecal glucocorticoid concentrations (fGCs) in gazelle ranged from 85 to $405 \mathrm{ng} \mathrm{g}^{-1}$, and when we tested for associations between $\mathrm{fT}$ and fGCs, we found a positive association between both hormones (least squares regression, $n=30, \log \mathrm{fT}$, coefficient $=0.33, \quad P=0.0048 ; \quad$ age,$\quad$ coefficient $=-0.01$, $P=0 \cdot 4405)$. We also tested for an association between testosterone and condition and found that $\mathrm{fT}$ was positively associated with hematocrit (HCT). With age included as a covariate in the model, this relationship was marginal (least squares regression, $n=31, \log \mathrm{fT}$, coefficient $=10 \cdot 38$, $P=0.0547$; age, coefficient $=-0 \cdot 11, \quad P=0.8294)$, but when the non-significant effect of age was dropped from the 
model, fT was significantly correlated with HCT $(n=34, \log$ fT, coefficient $=9 \cdot 85, P=0 \cdot 0465$ ).

\section{TESTOSTERONE, IMMUNITY AND PARASITE INFECTION}

To examine possible direct and indirect effects of testosterone on patterns of immunity and infection, we tested the effects of testosterone on immune function and parasite load either alone or in conjunction with GCs and other covariates including HCT, blood cell counts or horn size. fT had a significant and positive effect on both non-cellular (plasma bacteria killing) and cellular (circulating neutrophils) components of innate immunity (Fig. 3a,b). The testosterone effect on bacteria killing did not change when we accounted for fGCs and HCT, but HCT emerged as an additional significant predictor of bacteria killing (least squares regression, Table 1). Similarly, the neutrophil effect remained significant after we accounted for fGCs and lymphocyte counts (Table 1). In direct contrast to the relationship between testosterone and innate immune components, fT was significantly and negatively associated with our measure of adaptive immunity (circulating lymphocytes; Fig. 3c). However, once we accounted for additional covariates, including neutrophil counts, this relationship disappeared (least squares regression, Table 1). Overall, when we compared levels of support for our direct vs. indirect immunity models, the direct model was the best supported model for plasma bacteria killing, whereas variation in cellular immune components was best explained by indirect models (Table 2).

Of the 35 individuals sampled for parasites, $35(100 \%)$ were infected with strongyle nematodes, 33 (94\%) were infected with Morph A, 24 were infected with Morph B (69\%), 31 were infected with Morph C (89\%), and 13 (37\%) were infected with Trichuris. There was no association between fT and strongyle nematode infection, and similarly, fGCs and horn size had no effect on strongyle egg counts (least squares regression, Table 1). Morph A larval counts were signifi- cantly and positively associated with fT, but when we accounted for the effects of fGCs and horn size, this effect was no longer significant (negative binomial GLM, Table 1). For Morph B, fT was significantly and negatively associated with larval counts (least squares regression, Table 1). This effect remained when we controlled for fGCs and horn size; horn size was significantly and positively correlated with Morph B larval counts in the full model (least squares regression, Table 1). There was no significant effect of fT, fGCs or horn size on Morph C larval shedding in any model (least squares regression, Table 1). Finally, fT was not a significant predictor of Trichuris infection status in the testosterone only model, but after accounting for fGCs and horn size, higher fT was associated with a significant increase in the probability of Trichuris infection, while larger horn size was associated with a significant decrease in infection probability (likelihood ratio test, Table 1). Comparing direct and indirect models for the three parasites for which testosterone showed evidence of an effect, we found that the indirect models for Morph A and Morph B were best supported, while the indirect and direct models for Trichuris had fairly equivalent support (Table 2).

\section{Discussion}

Testosterone has been referred to as a 'double-edged sword', promoting male traits that enhance reproductive success on the one hand, but carrying costs of suppressed immunity and increased parasitism on the other hand (Folstad \& Karter 1992; Muehlenbein \& Bribiescas 2005). In free-ranging male Grant's gazelle, we found that testosterone was positively associated with secondary sexual traits and future resourceholding potential; however, the links between testosterone, immune function and parasitism were more complex. In fact, in direct contrast to predictions that testosterone should suppress immunity, the relationship between endogenous testosterone and immune function in free-ranging male gazelle was positive for some aspects of immunity. Furthermore,
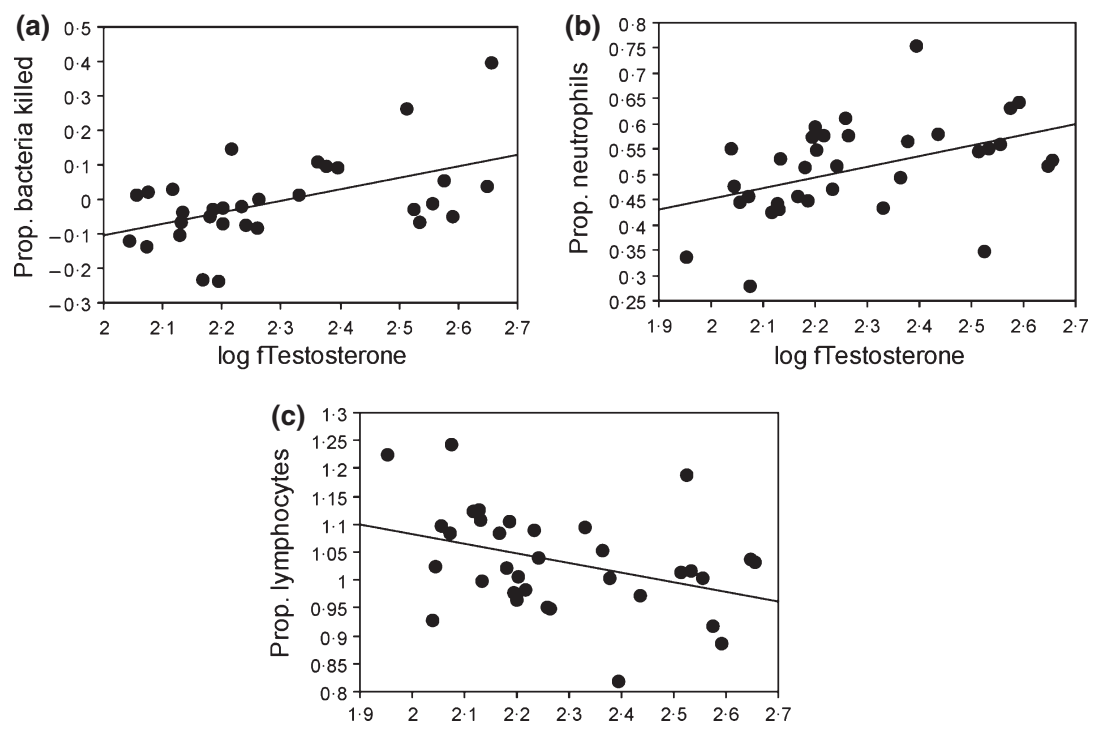

Fig. 3. Associations between faecal testosterone and measures of innate immunity: (a) plasma bacteria-killing ability, (b) proportion of circulating neutrophils (arcsin-transformed); and adaptive immunity: (c) proportion of circulating lymphocytes (arcsintransformed). 
Table 1. Effect of testosterone (fT) alone, or in combination with glucocorticoids (fGCs), condition and horn size, on measures of immunity and parasitism

\begin{tabular}{|c|c|c|c|c|c|c|c|c|c|c|c|}
\hline \multirow[b]{2}{*}{ Variable } & \multirow[b]{2}{*}{ Model type } & \multicolumn{4}{|c|}{ Testosterone only models } & \multicolumn{6}{|c|}{ Testosterone + models } \\
\hline & & $n$ & $\mathrm{fT}$ & Age & $T$ effect & $n$ & $\mathrm{fT}$ & Age & fGCs & $\operatorname{cov} \ddagger$ & $T$ effect \\
\hline \multicolumn{12}{|l|}{ Immunity } \\
\hline Plasma bacteria killing§ & Least squares regression & 21 & $0 \cdot 44^{* *}$ & $0 \cdot 009$ & + & 20 & $0 \cdot 31^{*}$ & $0 \cdot 01$ & $0 \cdot 08$ & $0 \cdot 01^{*}$ & + \\
\hline Neutrophils & Least squares regression & 31 & $0 \cdot 21^{* *}$ & $0 \cdot 009$ & + & 30 & $0.05^{*}$ & $-0 \cdot 002$ & $0 \cdot 007$ & $-0 \cdot 93 * * *$ & + \\
\hline Lymphocytes & Least squares regression & 31 & $-0 \cdot 17^{*}$ & $-0 \cdot 012 \dagger$ & - & 30 & $0 \cdot 03$ & $-0 \cdot 002$ & $-0 \cdot 02$ & $-0.99 * * *$ & 0 \\
\hline \multicolumn{12}{|l|}{ Parasites } \\
\hline Strongyle nematodes & Least squares regression & 31 & $0 \cdot 06$ & $0 \cdot 04$ & 0 & 30 & $0 \cdot 21$ & $0 \cdot 04$ & $-0 \cdot 01$ & $-0 \cdot 05$ & 0 \\
\hline Morph A & Negative binomial GLM & 31 & $2 \cdot 46^{*}$ & $0 \cdot 15$ & + & 30 & $2 \cdot 53 \dagger$ & $0 \cdot 09$ & $-0 \cdot 60$ & $-0 \cdot 02$ & $\mathrm{M}+$ \\
\hline Morph B & Least squares regression & 31 & $-2 \cdot 17^{*}$ & $0 \cdot 16$ & - & 30 & $-3 \cdot 82^{* *}$ & $0 \cdot 19^{*}$ & $-0 \cdot 72$ & $0 \cdot 67 * * *$ & - \\
\hline Morph C & Least squares regression & 31 & $0 \cdot 67$ & $0 \cdot 02$ & 0 & 30 & $0 \cdot 58$ & $0 \cdot 009$ & $0 \cdot 26$ & $-0 \cdot 009$ & 0 \\
\hline Trichuris & Likelihood ratio test $\mathbb{I}$ & 31 & $1 \cdot 49$ & $0 \cdot 2$ & 0 & 30 & $4 \cdot 22 *$ & $0 \cdot 88$ & $0 \cdot 29$ & $3.94 *(-)$ & + \\
\hline
\end{tabular}

Significance codes: $* * *<0 \cdot 001, * *<0 \cdot 01, *<0 \cdot 05, \dagger<0 \cdot 1$. Testosterone effects: + (positive), - (negative), 0 (no effect), $\mathrm{M}+($ marginal positive).

All values represent model coefficients.

$\$$ Cov indicates additional covariates in each model: plasma bacteria-killing model $=$ haematocrit; neutrophil model $=$ lymphocytes; lymphocyte model $=$ neutrophils; parasite models $=$ horn size.

§lasma bacteria-killing models also include handling time, and model coefficients for handling time are: testosterone only model $=-0 \cdot 006^{+}$; testosterone + model $=-0 \cdot 003$.

ILikelihood ratio test statistic $=\chi^{2}$.

relationships between testosterone and parasite infection ranged from negative to positive and were influenced by other factors, highlighting the potential for testosterone to affect parasite infection risk in diverse ways and via both direct and indirect pathways. Thus, while our data support the general notion that testosterone may underlie links between reproductive behaviour and parasite infection in male gazelle, it is clear that these links involve complex interactions among behavioural, immunological and endocrine components.

Unravelling the potential parasite-mediated fitness effects of testosterone requires a focus not only on the costs of testosterone with respect to immunity and infection but also on its relative benefits in terms of mating success. For free-living males to experience the obligatory trade-off between sexual signalling and immunity that underlies the ICHH (Folstad \& Karter 1992), natural levels of testosterone that generate variability in sexual signal intensity and/or mating behaviour should also be sufficient to induce immunity-related costs. For Grant's gazelle and other polygynous ungulates with resource defence mating systems, the acquisition of a territory or 'resource' is critical for attracting mates (Walther, Mungall \& Grau 1983; Vanpe et al. 2007). In these systems, males compete for resources, and horns and antlers play a central role in assessment and intrasexual competition (Hoem et al. 2007). Our composite measure of horn size was positively correlated with endogenous testosterone levels in male gazelle. In male ungulates, testosterone has been shown to promote horn and antler growth before puberty and during reproductively inactive periods, and also to suppress growth during bouts of active breeding (Lincoln 1998; Toledano-Diaz et al. 2007). The positive association we found between testosterone and horn size in gazelle may therefore reflect a link between current testosterone status and past sustained testosterone secretion that contributed to horn development. We also found

Table 2. Comparison of direct and indirect models of testosterone vs. immunity and parasitism

\begin{tabular}{|c|c|c|c|c|c|c|}
\hline Variable† & Model & Model type & $K$ & $\mathrm{AICc}$ & $\Delta \mathrm{AICc}$ & Akaike weight \\
\hline \multirow[t]{2}{*}{ Plasma bacteria killing } & $\mathrm{fT}+$ age + handling time & Direct & 5 & $-23 \cdot 0$ & 0 & 0.93 \\
\hline & $\mathrm{fT}+$ age + handling time + fGC + het & Indirect & 7 & $-17 \cdot 8$ & $5 \cdot 24$ & $0 \cdot 07$ \\
\hline \multirow[t]{2}{*}{ Neutrophils } & $\mathrm{fT}+$ age + fGC + lymphocytes & Indirect & 6 & $-144 \cdot 0$ & 0 & 1 \\
\hline & $\mathrm{fT}+$ age & Direct & 4 & $-72 \cdot 9$ & $71 \cdot 1$ & $<0 \cdot 0001$ \\
\hline \multirow[t]{2}{*}{ Lymphocytes } & $\mathrm{fT}+$ age $+\mathrm{fGC}+$ neutrophils & Indirect & 6 & $-141 \cdot 9$ & 0 & 1 \\
\hline & $\mathrm{fT}+$ age & Direct & 4 & $-70 \cdot 3$ & $71 \cdot 6$ & $<0.0001$ \\
\hline \multirow[t]{2}{*}{ Morph A } & $\mathrm{fT}+$ age $+\mathrm{fGC}+$ horn size & Indirect & 6 & $520 \cdot 3$ & 0 & 0.98 \\
\hline & $\mathrm{fT}+$ age & Direct & 4 & $527 \cdot 7$ & $7 \cdot 4$ & $0 \cdot 02$ \\
\hline \multirow[t]{2}{*}{ Morph B } & $\mathrm{fT}+$ age $+\mathrm{fGC}+$ horn size & Indirect & 6 & $89 \cdot 1$ & 0 & 0.997 \\
\hline & $\mathrm{fT}+$ age & Direct & 4 & $100 \cdot 9$ & $11 \cdot 8$ & $0 \cdot 003$ \\
\hline \multirow[t]{2}{*}{ Trichuris } & $\mathrm{fT}+$ age & Direct & 4 & $46 \cdot 73$ & 0 & $0 \cdot 57$ \\
\hline & $\mathrm{fT}+$ age + fGC + horn size & Indirect & 6 & $47 \cdot 32$ & $0 \cdot 59$ & $0 \cdot 43$ \\
\hline
\end{tabular}

$\dagger$ Includes only variables for which testosterone showed significant effects in either the direct or indirect model or both (see Table 1).

fT, faecal testosterone; fGCs, faecal glucocorticoid metabolites. 
that testosterone levels predicted male resource-holding potential, quantified as the frequency of territoriality. The result that males with higher levels of testosterone at capture were more likely to be territorial during the following 12 months strongly suggests that the testosterone levels we measured in our study were sufficient to promote variation in male behaviour and mating success. As such, it appears that naturally elevated testosterone levels in this system are likely associated with enhanced reproduction.

While the benefits of testosterone in terms of increased reproductive success are rarely questioned, its costs related to suppressed immune function and increased parasitism are a subject of extensive debate (Owen-Ashley, Hasselquist \& Wingfield 2004; Roberts, Buchanan \& Evans 2004; Roberts et al. 2007; Fuxjager et al. 2011). In our case, we found that endogenous testosterone was negatively associated with an index of standing adaptive immunity (lymphocyte counts) as predicted under the $\mathrm{ICHH}$, but testosterone was positively correlated with an index of innate immunity (neutrophil counts), suggesting that the effects of testosterone are not universally suppressive. Moreover, when we accounted for important covariates, including the negative correlation between white blood cell types (neutrophils and lymphocytes), the strong positive association between testosterone and neutrophils remained, while the negative association with lymphocytes disappeared, suggesting that the stronger relationship between immunity and testosterone in this system was one of potential enhancement rather than suppression.

The positive association between testosterone and innate immunity was also supported by a correlation between testosterone and plasma bacteria killing, an index of non-cellular, complement-mediated innate immunity (Matson, Tieleman $\&$ Klasing 2006). Relatively few studies have examined relationships between innate immunity and testosterone or other androgens (Greives et al. 2006; Kurtz et al. 2007; Ruiz et al. 2010). While one study found that endogenous concentrations of plasma testosterone were negatively associated with two distinct components of innate immunity in wild birds (Greives et al. 2006), an experimental study of lizards that manipulated both testosterone and food availability found that food supplementation increased testosterone and testosterone enhanced innate immunity (measured as plasma bacteria killing) in food-supplemented animals (Ruiz et al. 2010). This latter result suggests that both positive and negative correlations between testosterone and immunity can arise in the wild as a consequence of variation in food and/or energy availability. In our gazelle system, for example, testosterone may be immunoenhancing if more dominant (i.e. territorial) males with higher testosterone levels are better able to shunt resources to certain components of immune defence because they either have better access to food, are in better condition or use resources more efficiently (Evans, Goldsmith \& Norris 2000; Blas et al. 2006; McGraw \& Ardia 2007; Roberts \& Peters 2009). In support, we found that faecal testosterone was positively correlated with HCT, a commonly used indicator of condition in mammals (Delgiudice et al. 1992). Because HCT levels directly influence oxygen storage and delivery to muscle tissue, positive associations between testosterone and HCT may be indicative of increased physical stamina and endurance in high testosterone males, as recently suggested for red deer (Cervus elaphus, Malo et al. 2009). As such, HCT -driven enhancement in metabolic efficiency could potentially contribute to increased investment in innate immunity with increasing testosterone. Indeed, when HCT was included as a predictor in our model for plasma bacteria killing, it was significantly and positively correlated with bacteria killing. However, our comparison of direct and indirect models for bacteria killing showed much stronger support for the testosterone only model, suggesting either that direct effects of testosterone are more relevant in terms of variation in this immune variable or that the HCT effects we observed reflect some other physiological or condition index not accounted for in the present study.

In addition to the potential role of condition in testosterone-immunity relationships, several studies suggest that observed immunosuppressive effects of testosterone may be largely driven by correlated changes in glucocorticoids (Evans, Goldsmith \& Norris 2000; Owen-Ashley, Hasselquist $\&$ Wingfield 2004). One of these studies found that experimentally elevated testosterone levels impaired male antibody responses in house sparrows (Passer domesticus), but once correlated changes in corticosterone were accounted for testosterone appeared to enhance rather than suppress immunity (Evans, Goldsmith \& Norris 2000). In our study, we found that faecal testosterone was positively correlated with GCs in gazelle, but there was no evidence that associations between testosterone and immunity were influenced by GCs. In fact, GCs were not correlated with any of the three measures of immunity we assayed in this study. However, because we saw a weak association between capture and handling time and at least one measure of immunity used in this study, we cannot rule out the possibility that acute stress caused by capture and handling, coupled with potential correlations between fGC levels and individual responses to acute stress, may have obscured any relationships between fGCs and immune components. Nevertheless, a recent experimental study evaluating the effects of testosterone and corticosterone on immune function in zebra finches (Taeniopygia guttata) found that antibody responses were highest among individuals with the highest concentrations of both hormones (Roberts et al. 2007). This interactive and apparent immunoenhancing effect of both hormones supports the idea that stress and testosterone may act to redistribute components of immunity rather than universally suppress them (Braude, Tang-Martinez \& Taylor 1999). In particular, the testosterone immunoredistribution hypothesis suggests that immune resources may be temporarily shifted to sites of potential injury in response to elevations in circulating testosterone levels. For example, increases in testosterone may promote resistance to cutaneous infection via increased migration of neutrophils that facilitate wound healing, while less relevant responses are temporarily decreased (Braude, Tang-Martinez \& Taylor 1999). More generally, increases in testosterone may cause the reallocation of immune defences toward 
first-line defences (i.e. innate immunity) and away from more secondary responses (i.e. adaptive immunity). In support of these ideas, our direct models showed that neutrophils (standing innate immunity) were strongly and positively associated with testosterone in gazelle, whereas lymphocytes (standing adaptive immunity) were negatively associated with testosterone. Interestingly, however, the lymphocyte model with the most support in our study (the indirect model) showed no evidence of a negative testosterone-lymphocyte association, highlighting the need for a better understanding of how testosterone interacts with different components of the immune system.

For experimental studies, the ICHH predicts that testosterone should be negatively correlated with immunity and positively correlated with parasitism. For non-experimental studies, the expectations of the ICHH are less clear. In the wild, males with high testosterone may suffer depressed immunity leading to a positive association between testosterone and parasitism, or males with high testosterone may have 'good genes' that allow them to resist infection despite the immunosuppressive effects of testosterone leading to a negative correlation between testosterone and parasite load or no association at all. More complicated still is the fact that testosterone may alter parasitism not only through effects on male susceptibility or resistance (i.e. via immune function) but also by altering male exposure to parasites. While observational studies alone are insufficient to clarify the mechanisms underlying associations between testosterone and parasite load, they are useful for reconstructing the links between endogenous testosterone, ecologically relevant parasite loads and alternative ecological pathways by which variation in parasite load may be generated. We used multivariate models to test the effect of testosterone on five distinct parasite types in the gazelle system, both in the presence and absence of alternative factors that may influence these relationships. GCs were included in models to account for potential effects of stress on parasite susceptibility, while horn size was included as a proxy of male behaviour that could affect parasite exposure. In the direct models, the effects of testosterone on parasites ranged from no effect for three parasite types (strongyles, Trichuris, Morph C) to a positive effect on Morph A, and a negative effect on Morph B. Accounting for GCs and horn size changed the association between testosterone and two of the five parasites. For Trichuris, the relationship changed from no association to positive, and for Morph A, the relationship changed from significantly positive to only marginally positive (see Table 1). Moreover, horn size showed independent and significant effects on two parasites, being strongly and negatively associated with Morph B, but positively associated with Trichuris. In general, our model comparisons showed that the indirect parasite models had stronger support, which points to the importance of other factors in mediating testosterone-parasite relationships.

To our knowledge, these analyses reflect one of few attempts to track variation in endogenous testosterone, GCs, behaviour and multiple parasites simultaneously in a freeranging mammal (see Muehlenbein 2006; Muehlenbein \&
Watts 2010; Setchell et al. 2010 for notable examples), and our results lead to some interesting insights. For example, many studies testing associations between secondary sexual traits and parasite load in males assume that this relationship is driven by a testosterone-mediated trade-off between signal intensity and immune function or condition, but here, we show that for some parasites, horn size had effects independent of and in addition to testosterone. We found that testosterone was negatively associated with the number of Morph B larvae shed by male gazelle, while horn size was positively associated with larval shedding. Interpreted in the light of our immunological findings, a plausible interpretation of this result could be that immunoenhancing effects of testosterone decrease susceptibility to Morph B, but the behaviour of high testosterone males (signified by horn size) simultaneously increases exposure. Although the nature of our data does not allow us to assign cause and effect, the links we have made between testosterone, secondary sexual traits, behaviour, condition, immunity and parasite load in this system allow us to begin reconstructing plausible pathways of interaction among these variables that can be used to develop targeted experimental tests of parasite-mediated sexual selection in the wild.

Another key insight from our analyses is that the relationship between testosterone and parasitism varies from one parasite type to the next. From the literature, it is clear that there is a lack of broad support for the expected positive association between testosterone and parasite load as proposed by the ICHH (Roberts, Buchanan \& Evans 2004). Taking the few ungulate studies that have explored these relationships as an example, results span from positive associations between testosterone and strongyle nematodes in Alpine ibex (Capra ibex, Decristophoris, von Hardenberg \& McElligott 2007) and Elaphostrongylus lungworms in red deer (Malo et al. 2009) to no association between testosterone and strongyles or Protostrongylus lungworms in white-tailed deer (Odocoileus virginianus, Ditchkoff et al. 2001) and bighorn sheep (Ovis canadensis, Pelletier et al. 2005), respectively. Given that we found all possible relationships between testosterone and parasites across five different parasite types in a single study system, conflicting results emerging from different studies are not surprising. In fact, by comparing castrated, sham-operated and testosterone-implanted male mountain spiny lizards (Sceloporus jarrovi), Fuxjager et al. 2011) recently demonstrated that testosterone had distinct effects on different parasites in a field setting, resulting in positive, negative and no associations between plasma testosterone and parasite load depending on the parasite in question. They speculate that these effects might come about because testosterone affects different components of immunity differently, an idea supported by the opposing testosterone-immunity relationships we found in gazelle. They also suggest that opposing effects of testosterone on parasites may arise via effects of testosterone on host behaviour and parasite exposure. Our data show that a proxy for male behaviour not only affected the relationship between testosterone and parasites but also had independent effects on parasite load. This result provides intriguing evidence of a key role for behaviour and associated variation in 
parasite exposure in driving associations between testosterone and parasite infection, yet most studies focus exclusively on the immunological aspect of the testosterone-parasite relationship.

Overall, our integrative approach to examining the links between testosterone and parasite infection in free-living male mammals provides some key insights for the Grant's gazelle system in particular, and for understanding the ICHH and parasite-mediated sexual selection in general. In gazelle, where previous work has described an association between male territoriality and parasite load for one group of parasites (Ezenwa 2004), synthesis of our current results suggests that testosterone may indeed contribute to this pattern because territoriality is likely associated with elevated levels of testosterone. However, the specific mechanisms underlying such an effect could range from the suppressive effects of testosterone on immune function to correlated changes in behaviour that influence parasite exposure. As such, future studies parsing out the degree to which physiological changes in susceptibility vs. behavioural changes in exposure underlie testosteroneassociated variation in parasitism will be critical for understanding the role testosterone plays in generating variation in male parasitism (e.g. Mougeot et al. 2005), and consequences for the evolution of male mating behaviour.

Although the benefits of testosterone in terms of enhanced resource-holding potential and male mating success seem clear for gazelle and for many other species, the costs of testosterone with respect to immunity and infection are still rather murky. The 'double-edge' of the testosterone sword arises because of a presumed universal negative effect of testosterone on immunity and parasite resistance. However, we found evidence for simultaneous and opposing effects of testosterone on both immune function and parasitism in a single system. As such, it seems possible that suppressive effects of testosterone on some components of immunity may be buffered by enhanced responses in other components, leading to no net cost in terms of parasite infection when multiple parasites are considered. Furthermore, because behaviours associated with testosterone can lead to differences in exposure to different parasites, and not all in a single direction, both positive and negative indirect effects of testosterone on parasitism also seem likely. As a consequence, costs associated with increases in one parasite could be offset by declines in another (Fuxjager et al. 2011). Crucially, this suggests that to understand the diseaserelated costs of testosterone and how parasites and infectious diseases contribute to the overall viability costs of this hormone, the net effects of testosterone on the overall parasite community harboured by individual males need to be considered. In this context, it is also important for manipulative and observational studies to be conducted side by side as costs need to be quantified with respect to naturally occurring levels of testosterone and ecologically relevant parasite challenges.

\section{Acknowledgements}

Animal captures were conducted by Frontier Helicopters, NZ and the Kenya Wildlife Service Game Capture Unit. We thank J. Ewoi, A. Durcik, S. Hauver,
J. Hooge, J. Loroo, W. Sarmento, A. Williams and V. Zero for assistance in the field, and the Mpala Research Center for logistical support. The Ezenwa and Altizer laboratory groups at the University of Georgia provided helpful comments on the manuscript. The Kenya Ministry of Science, Education and Technology and the Kenya Wildlife Service gave permission to conduct this research in Kenya. Animal protocols used in this study were approved by the University of Montana Animal Care and Use Committee (\#023-09VEDBS-051509). The gazelle ACTH challenge was facilitated by Nadine Lamberski, Randy Rieches and the San Diego Wild Animal Park, and approved by the University of Montana (\#053-06VEDBS-102506) and Zoological Society of San Diego ACUC. The study was funded by a National Science Foundation grant (IOS-1101836) to V. Ezenwa. S. Ekernas was partially supported by the Montana Ecology of Infectious Disease NSF IGERT program.

\section{References}

Alonso-Alvarez, C., Bertrand, S. \& Sorci, G. (2007) Energetic reserves, leptin and testosterone: a refinement of the immunocompetence handicap hypothesis. Biology Letters, 3, 271-274.

Alonso-Alvarez, C., Bertrand, S., Faivre, B., Chastel, O. \& Sorci, G. (2007) Testosterone and oxidative stress: the oxidation handicap hypothesis. Proceedings Of The Royal Society B-Biological Sciences, 274, 819-825.

Altizer, S., Nunn, C.L., Thrall, P.H., Gittleman, J.L., Antonovics, J., Cunningham, A.A., Dobson, A.P., Ezenwa, V., Jones, K.E., Pedersen, A.B., Poss, M. \& Pulliam, J.R.C. (2003) Social organization and parasite risk in mammals: integrating theory and empirical studies. Annual Review of Ecology, Evolution and Systematics, 34, 517-547.

Bilbo, S.D. \& Nelson, R.J. (2001) Sex steroid hormones enhance immune function in male and female Siberian hamsters. American Journal of Physiology-Regulatory Integrative and Comparative Physiology, 280, R207R213.

Blas, J., Perez-Rodriguez, L., Bortolotti, G.R., Vinuela, J. \& Marchant, T.A. (2006) Testosterone increases bioavailability of carotenoids: insights into the honesty of sexual signaling. Proceedings of the National Academy of Sciences of the United States of America, 103, 18633-18637.

Bortolotti, G.R., Mougeot, F., Martinez-Padilla, J., Webster, L.M.I. \& Piertney, S.B. (2009) Physiological stress mediates the honesty of social signals. PLOSONE, 4, e4983.

Bowman, D.D. (1999) Georgis' Parasitology for Veterinarians, 7th edn. WB Saunders, Philadelphia.

Braude, S., Tang-Martinez, Z. \& Taylor, G.T. (1999) Stress, testosterone, and the immunoredistribution hypothesis. Behavioral Ecology, 10, 345-350.

Buchanan, K.L., Evans, M.R. \& Goldsmith, A.R. (2003) Testosterone, dominance signalling and immunosuppression in the house sparrow, Passer domesticus. Behavioral Ecology and Sociobiology, 55, 50-59.

Burnham, K.P. \& Anderson, D.R. (2002) Model Selection and Multimodel Inference: A Practical Information-Theoretic Approach. 2nd edn. SpringerVerlag, New York.

Casto, J.M., Nolan, V. \& Ketterson, E.D. (2001) Steroid hormones and immune function: experimental studies in wild and captive dark-eyed juncos (Junco hyemalis). American Naturalist, 157, 408-420.

Chandler, C.R., Ketterson, E.D., Nolan, V. \& Ziegenfus, C. (1994) Effects of testosterone on spatial activity in free-ranging male dark-eyed Juncos, Junco Hyemalis. Animal Behaviour, 47, 1445-1455.

Creel, S., Winnie, J.A. \& Christianson, D. (2009) Glucocorticoid stress hormones and the effect of predation risk on elk reproduction. Proceedings of the National Academy of Sciences of the United States of America, 106, $12388-12393$.

Decristophoris, P.M.A., von Hardenberg, A. \& McElligott, A.G. (2007) Testosterone is positively related to the output of nematode eggs in male Alpine ibex (Capra ibex) faeces. Evolutionary Ecology Research, 9, 1277-1292.

Delgiudice, G.D., Mech, L.D., Kunkel, K.E., Gese, E.M. \& Seal, U.S. (1992) Seasonal patterns of weight, hematology, and serum characteristics of freeranging female white-tailed deer in Minnesota. Canadian Journal Of Zoology-Revue Canadienne De Zoologie, 70, 974-983.

Denardo, D.F. \& Sinervo, B. (1994) Effects of steroid-hormone interaction on activity and home-range size of male lizards. Hormones and Behavior, 28, 273-287.

Ditchkoff, S.S., Lochmiller, R.L., Masters, R.E., Hoofer, S.R. \& Van Den Bussche, R.A. (2001) Major-histocompatibility-complex-associated variation in secondary sexual traits of white-tailed deer (Odocoileus virginianus): evidence for good-genes advertisement. Evolution, 55, 616-625.

Duckworth, R.A., Mendonca, M.T. \& Hill, G.E. (2001) A condition dependent link between testosterone and disease resistance in the house finch. 
Proceedings Of The Roval Society Of London Series B-Biological Sciences, 268, 2467-2472.

Evans, M.R., Goldsmith, A.R. \& Norris, S.R.A. (2000) The effects of testosterone on antibody production and plumage coloration in male house sparrows (Passer domesticus). Behavioral Ecology and Sociobiology, 47, $156-163$.

Ezenwa, V.O. (2003a) Habitat overlap and gastrointestinal parasitism in sympatric African bovids. Parasitology, 126, 379-388.

Ezenwa, V.O. (2003b) The effects of time of day on the prevalence of coccidian oocysts in antelope faecal samples. African Journal of Ecology, 41, 192-193.

Ezenwa, V.O. (2004) Host social behavior and parasitic infection: a multifactorial approach. Behavioral Ecology, 15, 446-454

Folstad, I. \& Karter, A.J. (1992) Parasites, bright males, and the immunocompetence handicap. American Naturalist, 139, 603-622.

Foreyt, W.J. (2001) Veterinary parasitology reference manual, 5th edn. Iowa State University Press, Ames, Iowa.

Forrester, S.G. \& Lankester, M.W. (1997) Extracting protostrongylid nematode larvae from ungulate feces. Journal of Wildlife Diseases, 33, 511-516.

Fuxjager, M.J., Foufopoulos, J., Diaz-Uriarte, R. \& Marler, C.A. (2011) Functionally opposing effects of testosterone on two different types of parasite: implications for the immunocompetence handicap hypothesis. Functional Ecology, 25, 132-138.

Gasbarre, L., Leighton, E. \& Sonstegard, T. (2001) Role of the bovine immune system and genome in resistance to gastrointestinal nematodes. Veterinary Parasitology, 98, 51-64.

Grear, D.A., Perkins, S.E. \& Hudson, P.J. (2009) Does elevated testosterone result in increased exposure and transmission of parasites? Ecology Letters, 12, 528-537.

Greives, T.J., Mcglothlin, J.W., Jawor, J.M., Demas, G.E. \& Ketterson, E.D (2006) Testosterone and innate immune function inversely covary in a wild population of breeding dark-eyed Juncos (Junco hyemalis). Functional Ecology, 20, 812-818.

Hamilton, W.D. \& Zuk, M. (1982) Heritable true fitness and bright birds: a role for parasites? Science, 218, 384-387.

Hasselquist, D., Marsh, J.A., Sherman, P.W. \& Wingfield, J.C. (1999) Is avian humoral immunocompetence suppressed by testosterone? Behavioral Ecology and Sociobiology, 45, 167-175.

Hoem, S.A., Melis, C., Linnell, J.D.C. \& Andersen, R. (2007) Fighting behaviour in territorial male roe deer Capreolus capreolus: the effects of antler size and residence. European Journal Of Wildlife Research, 53, 1-8.

Kurtz, J., Kalbe, M., Langefors, S., Mayer, I., Milinski, M. \& Hasselquist, D. (2007) An experimental test of the immunocompetence handicap hypothesis in a teleost fish: 11-ketotestosterone suppresses innate immunity in threespined sticklebacks. American Naturalist, 170, 509-519.

Lincoln, G.A. (1998) Reproductive seasonality and maturation throughout the complete life-cycle in the mouflon ram (Ovis musimon). Animal Reproduction Science, 53, 87-105.

Malo, A.F., Roldan, E.R.S., Garde, J.J., Soler, A.J., Vicente, J., Gortazar, C. \& Gomendio, M. (2009) What does testosterone do for red deer males? Proceedings Of The Royal Society B-Biological Sciences, 276, 971980.

Matson, K.D., Tieleman, B.I. \& Klasing, K.C. (2006) Capture stress and the bactericidal competence of blood and plasma in five species of tropical birds. Physiological and Biochemical Zoology, 79, 556-564.

McGraw, K.J. \& Ardia, D.R. (2007) Do carotenoids buffer testosteroneinduced immunosuppression? An experimental test in a colourful songbird Biology Letters, 3, 375-378.

Miles, D.B., Sinervo, B., Hazard, L.C., Svensson, E.I. \& Costa, D. (2007) Relating endocrinology, physiology and behaviour using species with alternative mating strategies. Functional Ecology, 21, 653-665.

Moller, A., Dufva, R. \& Allander, K. (1993) Parasites and the evolution of host social behavior. Advances in the Study of Behavior, 22, 65-102.

Mougeot, F., Redpath, S.M., Piertney, S.B. \& Hudson, P.J. (2005) Separating behavioral and physiological mechanisms in testosterone-mediated tradeoffs. American Naturalist, 166, 158-168.

Mougeot, F., Perez-Rodriguez, L., Martinez-Padilla, J., Leckie, F. \& Redpath, S.M. (2007) Parasites, testosterone and honest carotenoid-based signalling of health. Functional Ecology, 21, 886-898.

Muehlenbein, M.P. (2006) Intestinal parasite infections and fecal steroid levels in wild chimpanzees. American Journal of Physical Anthropology, 130, 546550 .

Muehlenbein, M.P. \& Bribiescas, R.G. (2005) Testosterone-mediated immune functions and male life histories. American Journal of Human Biology, 17, $527-558$.
Muehlenbein, M. \& Watts, D. (2010) The costs of dominance: testosterone, cortisol and intestinal parasites in wild male chimpanzees. BioPsychoSocial Medicine, 4, 21.

O'Brien, R.M. (2007) A caution regarding rules of thumb for variance inflation factors. Quality \& Quantity, 41, 673-690.

Owen-Ashley, N.T., Hasselquist, D. \& Wingfield, J.C. (2004) Androgens and the immunocompetence handicap hypothesis: unraveling direct and indirect pathways of immunosuppression in song sparrows. American Naturalist, 164, 490-505.

Pelletier, F., Page, K.A., Ostiguy, T. \& Festa-Bianchet, M. (2005) Fecal counts of lungworm larvae and reproductive effort in bighorn sheep, Ovis canadensis. Oikos, 110, 473-480.

Peters, A. (2000) Testosterone treatment is immunosuppressive in superb fairywrens, yet free-living males with high testosterone are more immunocompetent. Proceedings Of The Royal Society Of London Series B-Biological Sciences, 267, 883-889.

Roberts, M.L., Buchanan, K.L. \& Evans, M.R. (2004) Testing the immunocompetence handicap hypothesis: a review of the evidence. Animal Behaviour, 68, 227-239.

Roberts, M. \& Peters, A. (2009) Is testosterone immunosuppressive in a condition-dependent manner? An experimental test in blue tits Journal of Experimental Biology, 212, 1811-1818.

Roberts, M.L., Buchanan, K.L., Hasselquist, D. \& Evans, M.R. (2007) Effects of testosterone and corticosterone on immunocompetence in the zebra finch. Hormones and Behavior, 51, 126-134.

Ruiz, M., French, S.S., Demas, G.E. \& Martins, E.P. (2010) Food supplementation and testosterone interact to influence reproductive behavior and immune function in Sceloporus graciosus. Hormones and Behavior, 57, 134 139.

Setchell, J.M., Smith, T., Wickings, E.J. \& Knapp, L.A. (2010) Stress, social behaviour, and secondary sexual traits in a male primate. Hormones and Behavior, 58, 720-728.

Stelfox, J.B., Hudson, R.J. \& Groer, N. (1985) Relationship among physical traits, age and social status in Thomson's and Grant's gazelle. Applied Animal Behavior Science, 13, 347-357.

Toledano-Diaz, A., Santiago-Moreno, J., Pulido-Pastor, A. \& Lopez-Sebastian, A. (2007) Horn growth related to testosterone secretion in two wild Mediterranean ruminant species: the Spanish ibex (Capra pyrenaica hispanica) and European mouflon (Ovis orientalis musimon). Animal Reproduction Science, 102, 300-307.

Trillmich, F., Rea, L., Castellini, M. \& Wolf, J.B.W. (2008) Age-related changes in hematocrit in the Galapagos sea lion (Zalophus wollebaeki) and the Weddell seal (Leptonychotes weddellii). Marine Mammal Science, 24, 303-314.

Vanpe, C., Gaillard, J.M., Kjellander, P., Mysterud, A., Magnien, P., Delorme, D., Van Laere, G., Klein, F., Liberg, O. \& Hewison, A.J.M. (2007) Antler size provides an honest signal of male phenotypic quality in roe deer. American Naturalist, 169, 481-493.

Villanua, D., Perez-Rodriguez, L., Gortazar, C., Hofle, U. \& Vinuela, J. (2006) Avoiding bias in parasite excretion estimates: the effect of sampling time and type of faeces. Parasitology, 133, 251-259.

Walther, F.R. (1972) Social grouping in Grant's gazelle (Gazella granti Brooke 1827) in the Serengeti national park. Zietschrift fur Tierpsychologie, 31, 348403.

Walther, F.R., Mungall, E.C. \& Grau, G.A. (1983) Gazelles and their Relatives. A Study in Territorial Behavior. Noyes Publications, Park Ridge.

Received 8 May 2011; accepted 6 September 2011

Handling Editor: Jennifer Grindstaff

\section{Supporting Information}

Additional supporting information may be found in the online version of this article.

Figure S1. Male status classification.

As a service to our authors and readers, this journal provides supporting information supplied by the authors. Such materials may be reorganized for online delivery, but are not copy-edited or typeset. Technical support issues arising from supporting information (other than missing files) should be addressed to the authors. 